\title{
Limits on 331 vector bosons from LHC proton collision data
}

\author{
A. A. Nepomuceno $\odot^{*}$ \\ Departamento de Ciências da Natureza, Universidade Federal Fluminense, \\ Rua Recife s/n, Rio das Ostras, Rio de Janeiro 28895-532, Brazil \\ B. Meirose $\odot^{\dagger}$ \\ Department of Physics, University of Texas at Dallas, \\ 800 West Campbell Road, Richardson, Texas 75080, USA
}

(Received 2 December 2019; accepted 23 January 2020; published 13 February 2020; corrected 26 June 2020)

In this paper, limits are set on bileptons masses and couplings in the context of a 331 model, as well as generic, non-331 models predicting bileptons. The following measurable processes are studied: $p p \rightarrow \ell^{+} \ell^{+} \ell^{-} \ell^{-} X, p p \rightarrow \ell^{+} \ell^{-} \nu \nu X$ and $p p \rightarrow \ell^{+} \ell^{-} X$. Experimental limits on singly charged bileptons masses and couplings within a 331 model are also obtained for the first time. With the results, an over 20 -year-old experimental limit on vector bileptons is increased by $60 \%$. The computed limits are now the most stringent ones for these particles.

DOI: 10.1103/PhysRevD.101.035017

\section{INTRODUCTION}

The extended elecroweak gauge symmetry group $S U(3)_{C} \otimes S U(3)_{L} \otimes U(1)_{X}$ is by now a nearly 30-yearold prediction [1-4]. In the absence of any reports of deviations from the Standard Model (SM), in particular by the LHC experimental collaborations, it is important to understand just how strong tensions between predictions from 331 models and experimental results are. This is important on several grounds. 331 models are based on the gauge symmetry $S U(3)_{C} \otimes S U(3)_{L} \otimes U(1)_{X}$ and as an $S U(N)$ type of group they predict the existence of $N^{2}-1=8$ gauge bosons in its $S U(3)_{L}$ sector. Three of the gauge bosons are the familiar ones from the SM, namely the positively and negatively charged $W^{ \pm}$bosons and the neutral $Z^{0}$ boson. The other five non-SM gauge bosons are the positively and negatively doubly charged bileptons $Y^{ \pm \pm}$, the positively and negatively singly charged bileptons $V^{ \pm}$, and the heavy neutral $Z^{\prime}$ boson. These electric charges of the exotic bosons are predicted in the particular version of the model we are considering, the Frampton-PisanoPleitez (FPP) 331 model [3,4], but other charges are possible in other 331 model versions. For a review of 331 models we refer the reader to Ref. [5].

\footnotetext{
*andrenepomuceno@id.uff.br

Bernhard.Meirose@cern.ch
}

Published by the American Physical Society under the terms of the Creative Commons Attribution 4.0 International license. Further distribution of this work must maintain attribution to the author(s) and the published article's title, journal citation, and DOI. Funded by SCOAP.
There are many interesting aspects of the FPP 331 model worth noticing [6] but arguably, the most intriguing one is the explanation of three quark-lepton families, which is one of the main theoretical motivations for expecting bileptons in Nature. This is accomplished via a nontrivial anomaly cancellation in the model that takes place between families, unlike in the SM where the desired anomaly-free condition is accomplished for each family separately.

Doubly charged bileptons are the most striking prediction of the FPP 331 model, but the expected LHC mass reach, considering the cleanest channel producing bileptons in proton-proton collisions, is $\sim 1 \mathrm{TeV}$ and even if one considers the High-Luminosity LHC (HL-LHC) project, formerly known as Super-LHC (sLHC), the reach increases only by $20 \%$ [6]. Due to kinematics, however, these conclusions are heavily dependent on the leptoquarks masses that are predicted in the fermionic sector of the model. Should these particles be at least as heavy as $2 \mathrm{TeV}$ the discovery reach for bileptons increases significantly [7]. Regarding experimental constraints, a mass bound of $M_{Y}>740 \mathrm{GeV}$ for doubly charged bilepton gauge bosons was derived from constraints on fermion pair production at LEP and leptonflavor violating charged lepton decays [8]. It represented the most useful limit on doubly charged vector bileptons for the past 20 years. Searches for muonium-antimuonium conversion [9] at PSI put the more stringent limit of $M_{Y}>850 \mathrm{GeV}$ on $Y^{ \pm \pm}$; however, this is a less general limit as it assumes flavor-diagonal coupling for bileptons. Constraints on singly charged bileptons are due to experimental limits on $\mu_{R} \rightarrow$ $e \nu \nu$ and $\nu_{\mu} \rightarrow \nu_{e}$ oscillations, and are about $40 \%$ lower than for doubly charged bileptons [10]. All these limits are now surpassed by the results of this article. 
The most stringent limits on $Z^{\prime}$ gauge bosons within 331 models up to now have been derived from weak charge data of Cesium and proton [11] and establish a lower bound of $4 \mathrm{TeV}$ for these particles in the minimal version of the 331 model, with a lowest value of $1.25 \mathrm{TeV}$ for a version of the 331 model discussed in [12]. Recent limits from the ATLAS Collaboration imply a $Z^{\prime}$ mass lower limit of $5.1 \mathrm{TeV}$ in the sequential Standard Model [13] at 95\% confidence level. While this is the most stringent limit on any $Z^{\prime}$ mass, it is not applicable to 331 models. As pointed out in Ref. [14], the lower bounds of $Z^{\prime}$ mass can be significantly lower than those obtained from LHC, if other decay channels of $Z^{\prime}$ into new particles are included. The limits obtained in our work are, therefore, the most stringent limits for $Z^{\prime}$ in a 331 model framework using hadron collision data.

This article is organized as follows. Section II describes in detail the numerical implementation of the studied processes in Monte Carlo event generators and in a fast parametric detector simulation, including an overall description of the masses and couplings used in the simulations. In Sec. III calculated cross sections at $13 \mathrm{TeV}$ center-of-mass energy, as well as widths for given particle masses, are described. The limit setting procedures using a Bayesian approach is discussed in Sec. IV, followed by the specific limits obtained for $Z^{\prime}$, doubly charged and singly charged bileptons in Secs. V, VI and VII, respectively. Conclusions are presented in Sec. VIII.

\section{NUMERICAL IMPLEMENTATION}

Bileptons can be produced in pairs at the LHC through a Drell-Yan-like process mediated by the photon, the SM $Z^{0}$ and the new neutral heavy boson $Z^{\prime}$. They can also be produced via a $t$-channel with a leptoquark exchange. The additional $t$-channels are needed in order to guarantee that all relevant quark subprocesses respect unitarity. The Feynman diagrams for bilepton production are shown in Fig. 1.

In doubly charged bilepton pair production, each bilepton decays into a same-sign lepton pair, and therefore the natural processes to search for these type of bileptons is $p p \rightarrow Y^{++} Y^{--} \rightarrow \ell^{+} \ell^{+} \ell^{-} \ell^{-} X$, where $\ell=e, \mu$. For singly charged bileptons, the characteristic decay is a signal with an opposite-charge lepton pair and two neutrinos, $p p \rightarrow V^{+} V^{-} \rightarrow \ell^{+} \ell^{-} \nu \nu X$. Leptonic decay is also the cleanest signal for $Z^{\prime}$ searches, hence in this work we investigate the processes $p p \rightarrow Z^{\prime} \rightarrow \ell^{+} \ell^{-} X$.

The production cross sections for the exotic bosons were calculated using the CALCHEP event generator [15], where the model was previously implemented [16]. Events were generated for all the processes mentioned above using the CTEQ6L1 parton distribution function and center-ofmass energy of $13 \mathrm{TeV}$. The generated events at parton level were processed by PYTHIA8 [17] to simulate parton shower, hadronization, and underlying event. A fast detector simulation was performed using DELPHES [18] with ATLAS
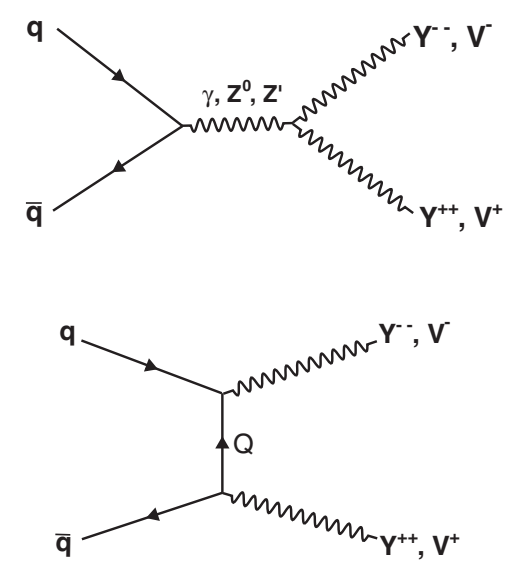

FIG. 1. Feynman diagrams for bileptons production at LHC. The $t$-channel production is mediated by a leptoquark.

detector configuration. Pileup is taken into account by overlaying minimum-bias events simulated with PYTHIA with the hard-scatter process. The average number of $p p$ interaction per bunch crossing considered in this work is 24 .

The bilepton masses were taken in the range of 400 to $1300 \mathrm{GeV}$, and the $Z^{\prime}$ mass ranged from 1 to $5 \mathrm{TeV}$. The leptoquarks masses were fixed at $M_{Q}=1.5 \mathrm{TeV}$. When generating the bilepton events, the $Z^{\prime}$ mass was fixed at $5 \mathrm{TeV}$. This particular choice of $M_{Z^{\prime}}$ has no significant impact on the bilepton cross section, since the on-shell $Z^{\prime}$ exchange contribution is only relevant for $M_{Z^{\prime}}<1 \mathrm{TeV}$. In order to perform a more general analysis, the coupling $g_{3 l}$ between bileptons and leptons is also varied as a free parameter, following a similar approach used in Refs. $[19,20]$. Besides the value $g_{3 l}=1.19 \sqrt{4 \pi \alpha}=0.373$, that characterizes a 331 model [21], five other possibilities are investigated: $g_{3 l}=2,4,6,8,10$.

\section{CROSS SECTIONS AND WIDTHS}

The width-mass ratio $(\Gamma / M)$ for bileptons is shown in Fig. 2. The ratio for three representative values of $g_{3 l}$ are plotted. For small values of $g_{3 l}$, the bilepton resonance is very narrow, with $\Gamma / M<1 \%$. For $g_{3 l}>2$, it increases rapidly, reaching $\sim 30 \%$ for $g_{3 l}=4$ and $\sim 190 \%$ for $g_{3 l}=10$. It can be noticed that, since the bilepton decays are the same for the interval shown, the ratio remains constant for each value of $g_{3 l}$.

Figure 3 shows the $\Gamma / M$ ratio for $Z_{331}^{\prime}$. The $Z^{\prime}$ is a broad resonance in the minimal 331 model. The $Z_{331}^{\prime}$ width becomes larger from $M_{Z_{331}^{\prime}}=3 \mathrm{TeV}$ as a result of the boson decay to the model's leptoquarks.

Figure 4 shows the cross section for four-lepton production mediated by bileptons as a function of $M_{Y}$, for different coupling values. The production of bilepton pairs does not depend on $g_{3 l}$, and the branching fractions $\operatorname{Br}\left(Y^{ \pm \pm} \rightarrow \ell^{ \pm} \ell^{ \pm}\right)$are not affected by $g_{3 l}$ variation. 


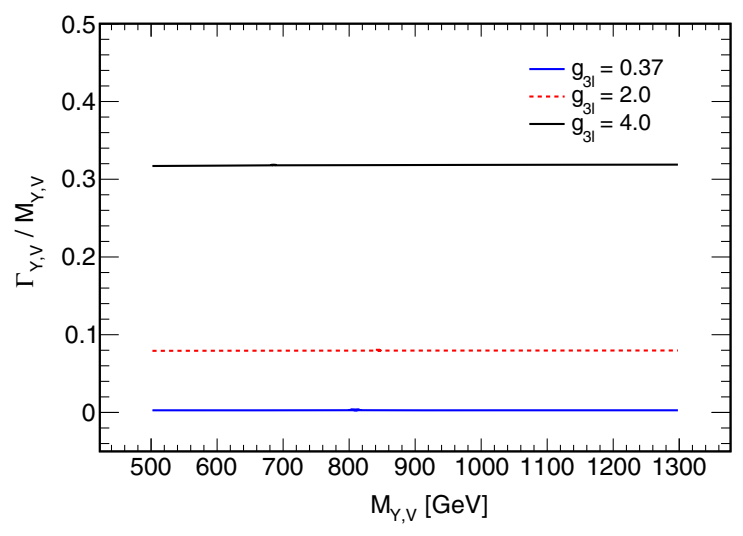

FIG. 2. Width-mass ratio as a function of mass for bileptons calculated for three values of $g_{3 l}$.

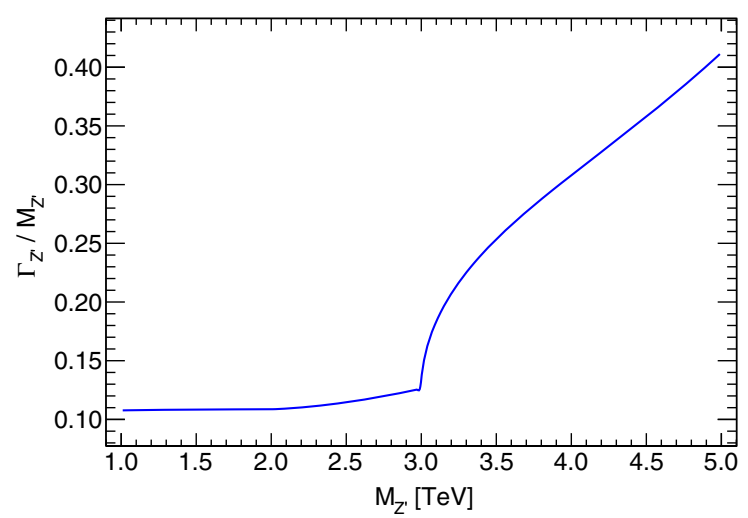

FIG. 3. Width-mass ratio as a function of $Z_{331}^{\prime}$ mass. From $M_{Z_{331}^{\prime}}=3 \mathrm{TeV}$, the decay $Z_{331}^{\prime} \rightarrow Q \bar{Q}$ becomes kinematically allowed.

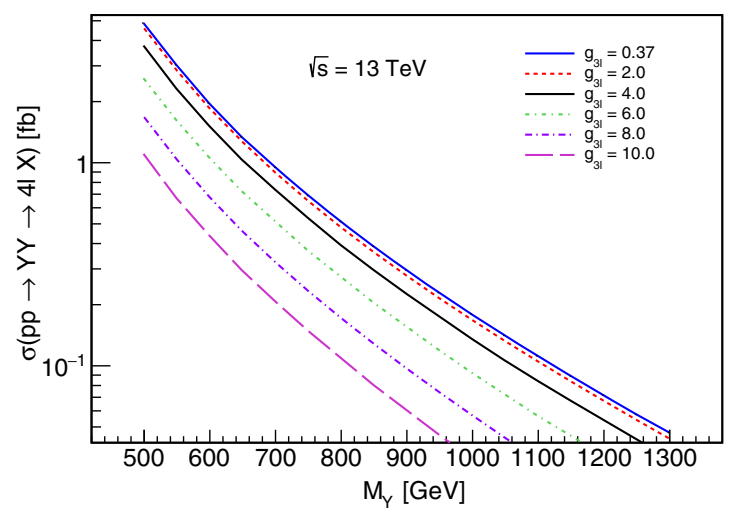

FIG. 4. LO cross section for four leptons production mediated by doubly or singly bileptons considering different values of $g_{3 l}$ at $\sqrt{s}=13 \mathrm{TeV}$.

The total bilepton width, however, increases as $g_{3 l}$ rises, causing the total cross section to decrease.

This cross-section behavior is characteristic for the case we are studying, where the bileptons decay only to leptons and not to leptoquarks $\left(M_{Q}>M_{Y, V}\right)$. The cross section for the process $p p \rightarrow V^{+} V^{-} \rightarrow \ell^{+} \ell^{-} \nu \nu X$ is the same as for $p p \rightarrow Y^{++} Y^{--} \rightarrow \ell^{+} \ell^{+} \ell^{-} \ell^{-} X$. The $\mathrm{LO}$ cross sections are multiplied by a $k$-factor of 1.25 to take into account NLO effects [22].

The dilepton production cross section mediated by $Z_{331}^{\prime}$ is shown by the solid red line of Fig. 6. The small bump around $3 \mathrm{TeV}$ indicates the point from where the exotic decay $Z_{331}^{\prime} \rightarrow Q \bar{Q}$ becomes kinematically allowed.

\section{LIMIT SETTING PROCEDURE AND VALIDATION}

The invariant mass distributions $m(\ell \ell)$ of lepton pairs derived from doubly charged bileptons and from $Z^{\prime}$ productions are used to calculate upper limits on $\sigma B$, where $\sigma$ is the cross section of the new physics process and $B$ is the branching ratio of $Z^{\prime}$ and $Y^{ \pm \pm}$decaying to leptons. For the singly charged bilepton analysis, another distribution, the so-called "stransverse" mass [23,24], is used. A likelihood function defined as the product of Poisson probabilities over all distribution's bins of a given search is constructed. To calculate the limits, a Bayesian approach is applied with a flat prior probability distribution for $\sigma B$, and the Bayes theorem is employed to evaluate the marginal posterior probability density function, $\mathcal{L}(\sigma B \mid N)$, where $N$ is the number of observed events. Upper limits at 95\% C.L. are set on $\sigma B$ by integrating the posterior probability density function as

$$
0.95=\frac{\int_{0}^{(\sigma B)_{u p}} \mathcal{L}(\sigma B \mid N) d(\sigma B)}{\int_{0}^{\infty} \mathcal{L}(\sigma B \mid N) d(\sigma B)}
$$

where $(\sigma B)_{u p}$ is the calculated limit. The calculation is performed with the Bayesian Analysis Tool Kit [25]. The upper limits on $\sigma B$ are translated into lower limits on the mass of the new bosons by using the theoretical cross section for the new boson production. The limits obtained with data are called observed limits. The expected limits are obtained by running a large number of pseudoexperiments where it is assumed that only background events are present. All the estimated backgrounds and efficiencies used in our analysis are extracted from the relevant ATLAS publications.

In order to validate the signals' simulation and the limit setting procedure described above, we calculate limits on $Z_{S S M}^{\prime}$ and $Z_{\chi}^{\prime}$ models, with the $Z^{\prime}$ signals simulated with PYTHIA and DELPHES, and compare the results with those obtained by the ATLAS Collaboration [26]. Table I shows the results for electron and muon channels combined. As we can see, there is a good agreement between the ATLAS results and the limits obtained using the signals simulated with DELPHES.

To set limits on bileptons masses and couplings, and on $Z^{\prime}$ mass, different ATLAS searches are considered. 
TABLE I. Comparison between the limits on two popular $Z^{\prime}$ models obtained by ATLAS Collaboration and by the PYTHIA and DELPHES simulation performed in this work.

\begin{tabular}{|c|c|c|c|c|}
\hline \multirow[b]{3}{*}{ Model } & \multicolumn{4}{|c|}{ Lower limits on $M_{Z^{\prime}}[\mathrm{TeV}]$} \\
\hline & \multicolumn{2}{|c|}{ ATLAS } & \multicolumn{2}{|c|}{ DELPHES } \\
\hline & observed & expected & observed & expected \\
\hline$\overline{Z_{S S M}^{\prime}}$ & 4.5 & 4.5 & 4.4 & 4.4 \\
\hline$Z_{\chi}^{\prime}$ & 4.1 & 4.0 & 4.0 & 4.0 \\
\hline
\end{tabular}

The data sample in all the analysis corresponds to an integrated luminosity of $36.1 \mathrm{fb}^{-1}$. These are discussed in the following (V-VI) sections.

\section{LIMITS ON $Z_{331}^{\prime}$}

Limits on $Z_{331}^{\prime}$ mass are set using the data from Ref. [26]. The signal candidates are selected by requiring at least one pair of same flavor leptons (electrons or muons). Electrons candidates are selected if they have transverse energy $\left(E_{T}\right)$ greater than $30 \mathrm{GeV}$ and pseudorapidity $|\eta|<2.47$. Events inside the region $1.37 \leq|\eta| \leq 1.52$ are excluded due to poor energy resolution. Muons candidates are required to have transverse momentum $\left(p_{T}\right)$ greater than $30 \mathrm{GeV}$, $|\eta|<2.5$ and opposite charges. The opposite charge requirement is not applied to the electron channel since it is not applied in data. If more than two leptons are found, the ones with highest transverse momentum are kept.

The inputs to the statistical analysis are the reconstructed invariant mass distribution of the selected events shown in Figs. 5(a) and 5(b) for electron and muon channels, respectively. The data points are shown with their statistical uncertainties. The dark histogram is the total estimated background. Two $Z_{331}^{\prime}$ signals with masses of 3 and $4 \mathrm{TeV}$ are also displayed for comparison.

The observed and expected upper limits $(\sigma B)_{u p}$ are calculated for various $Z_{331}^{\prime}$ mass hypothesis and the results are shown in Fig. 6. The lower limits on $M_{Z_{331}^{\prime}}$ are extracted from the crossing point between the theoretical cross section and $(\sigma B)_{u p}$. The observed and expected mass limits found are 3.7 and $3.6_{-0.2}^{+0.1} \mathrm{TeV}$, respectively. These limits represent significant improvement compared to previous bounds on $Z^{\prime}$ from 331 models [27-29].

\section{LIMITS ON DOUBLY CHARGED BILEPTONS}

In this section, the ATLAS search for doubly charged Higgs bosons [30] are interpreted in terms of doubly charged bileptons. The analysis focuses on $Y^{ \pm \pm} \rightarrow e^{ \pm} e^{ \pm}$ and $Y^{ \pm \pm} \rightarrow \mu^{ \pm} \mu^{ \pm}$decay channels. The bilepton decay in different lepton flavors, $Y^{ \pm \pm} \rightarrow e^{ \pm} \mu^{ \pm}$, is not considered since there is no usable data available for this particular channel.

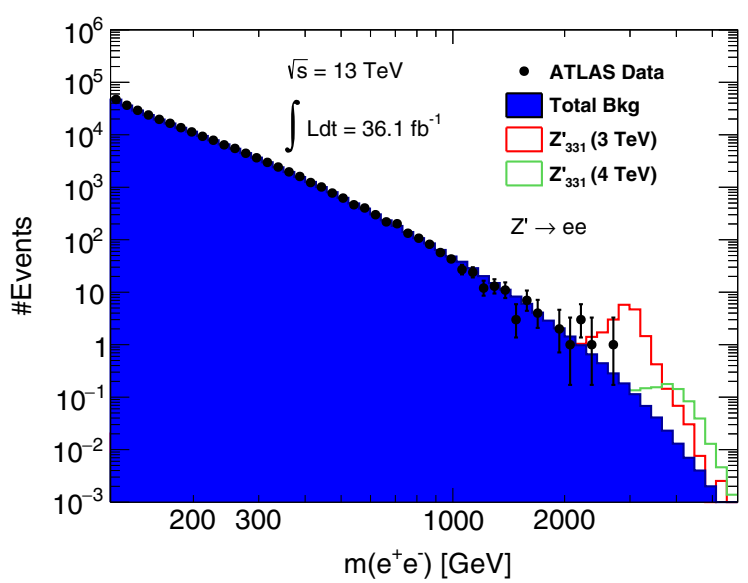

(a)

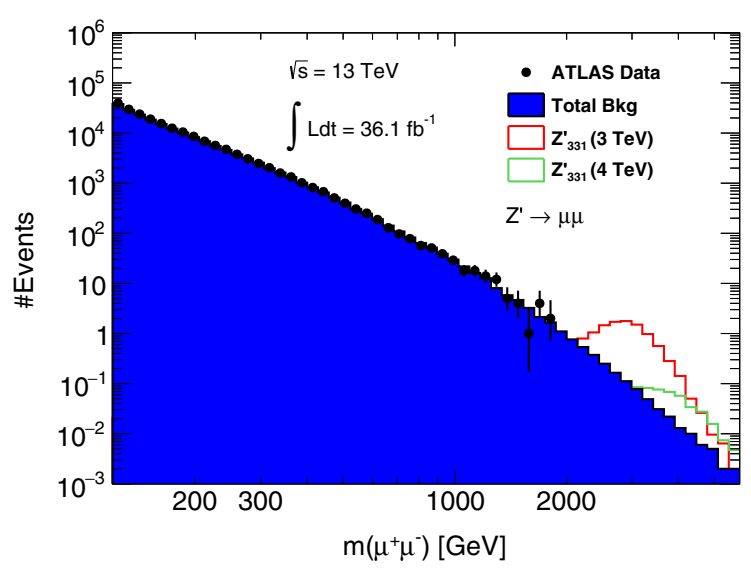

(b)

FIG. 5. Dilepton invariant mass distribution for electron (a) and muon (b) channels. The black points show the ATLAS data with the statistical uncertainties. The dark histogram is the total background, and the red and green open histograms are the distributions for two $Z_{331}^{\prime}$ mass hypotheses.

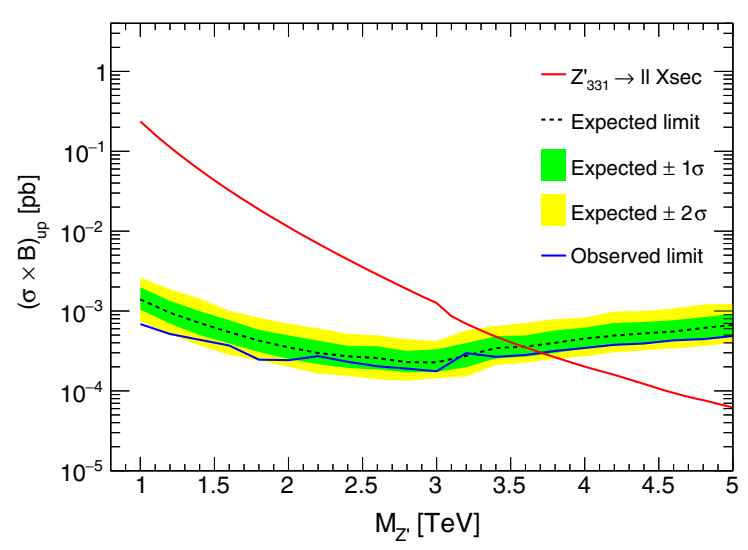

FIG. 6. Observed and expected limits on $\sigma B$ as a function of the $Z_{331}^{\prime}$ mass assumption. The green and yellow bands show the $1 \sigma$ and $2 \sigma$ variation on the expected limit, respectively. The solid red line is the theoretical cross section for dilepton production mediated by $Z_{331}^{\prime}$. 
As the doubly charged bileptons are produced in pairs, the selected final state must have at least three same flavor leptons, all within the inner detector coverage $(|\eta|<2.5)$. Events with three leptons are required to have one samesign lepton pair and one lepton of opposite charge $\left(\ell^{ \pm} \ell^{ \pm}\right.$ $\left.\ell^{\mp}\right)$. If there are four leptons in the event, the net electric charge must be zero. Following the same selections applied to data, events with at least one $b$-tagged jet are rejected to suppress background from top quarks. An event is also rejected if the invariant mass of the opposite charge same flavor leptons pair is in the range $82.1 \mathrm{GeV}<$ $m\left(\ell^{+} \ell^{-}\right)<101.2 \mathrm{GeV}$. In the three leptons events, signal sensitivity is optimized by imposing the same charge lepton separation to be $\Delta R\left(\ell^{ \pm} \ell^{ \pm}\right)>3.5$ and their combined transverse momentum to be $p_{T}\left(\ell^{ \pm} \ell^{ \pm}\right)>100 \mathrm{GeV}$. In addition, the scalar sum of the individual leptons transverse momenta is required to be greater than $300 \mathrm{GeV}$.

The invariant mass of the same sign leptons pair is used as discriminant variable. In the case of events with four leptons, the variable considered is the average invariant mass of the two same charge leptons:

$$
\bar{m} \equiv \frac{m\left(\ell^{+} \ell^{+}\right)+m\left(\ell^{-} \ell^{-}\right)}{2} .
$$

The analysis is performed in the region where $m\left(\ell^{ \pm} \ell^{ \pm}\right)$ and $\bar{m}$ are both above $200 \mathrm{GeV}$. Figures 7(a) and 7(b) show the invariant mass for three and four leptons events combined, in electron and muon channels. Along with the ATLAS data and background, two bilepton signals are shown. The hatched bands represent the systematic uncertainty on the background.

Limits are set considering different bilepton mass and coupling $\left(g_{3 l}\right)$ hypotheses. The excluded parameter space is shown in Fig. 8, from where we conclude that doubly charged bileptons with masses between $\sim 750$ and $\sim 1200 \mathrm{GeV}$ are excluded. For high values of $g_{3 l}$, the observed limits are weaker than the expected due to the larger bilepton width. It it interesting to note that the four leptons production cross section does not change significantly for $g_{3 l}<1$, which means that $M_{Y}>1.2 \mathrm{TeV}$ is the maximum bound on doubly charged bileptons mass that can be reached with the data considered.

\section{LIMITS ON SINGLY CHARGED BILEPTONS}

The production of singly charged bileptons are characterized by events with two leptons and missing transverse energy in the final state. It is the same measurable final state studied in charginos and sleptons searches from supersymmetric models. Here we use the ATLAS data from supersymmetric searches [31] to set bounds on singly charged bileptons. This analysis considers all possible leptonic final states produced by the singly charged

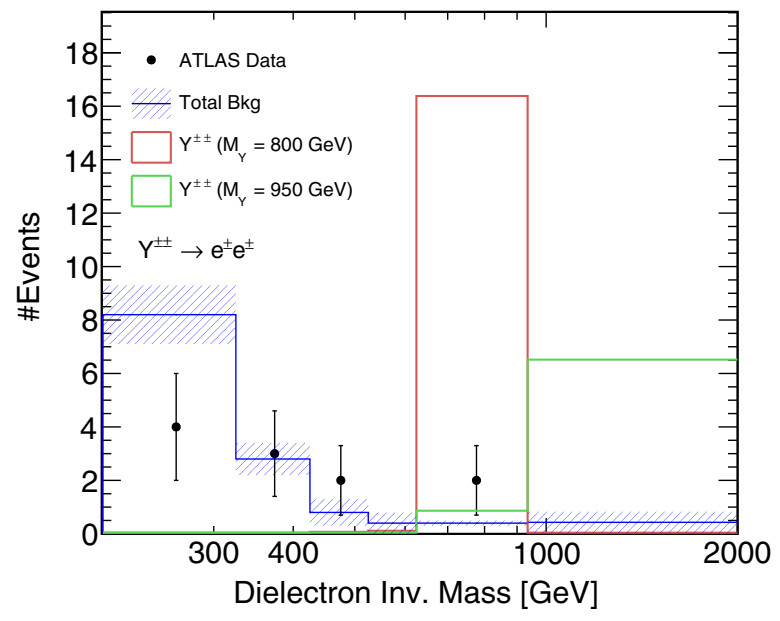

(a)

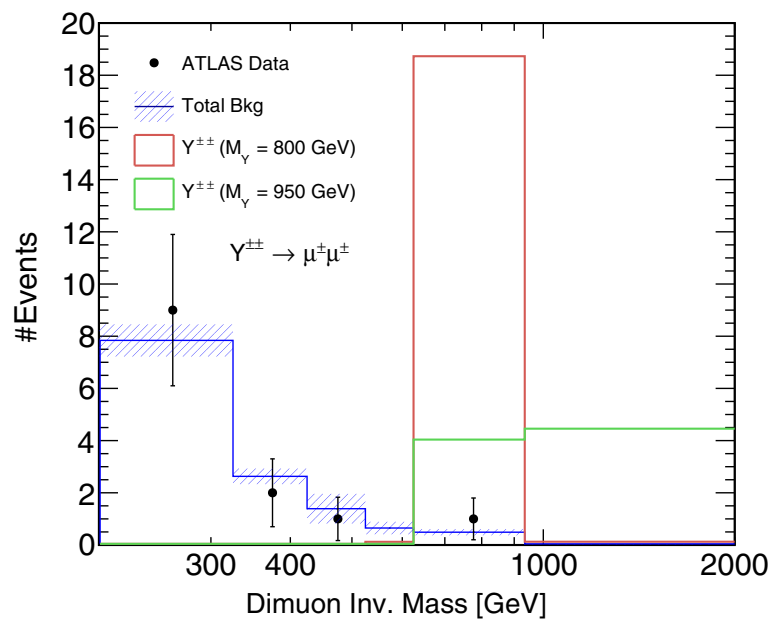

(b)

FIG. 7. Invariant mass distribution for same-charge electrons (a) and same-charge muons (b) pairs. The hatched band represents the total systematic uncertainty on the background determined by ATLAS. Two doubly bileptons signals of masses 800 and $950 \mathrm{GeV}$ are shown.

bilepton pair production: $V^{ \pm} \rightarrow e^{+} \nu e^{-} \nu, V^{ \pm} \rightarrow \mu^{+} \nu \mu^{-} \nu$, $V^{ \pm} \rightarrow e^{+} \nu \mu^{-} \nu$ and $V^{ \pm} \rightarrow \mu^{+} \nu e^{-} \nu$.

The signal events candidates are required to have exactly two leptons and missing transverse energy. The leptons can be of the same or different flavors, but they must have opposite charges. Additionally, leptons are required to have $|\eta|<2.5$ and $p_{T}>10 \mathrm{GeV}$, and a dilepton invariant mass of $m_{\ell \ell}>110 \mathrm{GeV}$. An event is rejected if there is a $b$-tagged jet with $p_{T}>20 \mathrm{GeV}$ or any other jet with $p_{T}>60 \mathrm{GeV}$. The selected events are separated in two categories: same-flavor $\left(e^{+} e^{-}\right.$and $\mu^{+} \mu^{-}$ events) and different-flavor ( $e^{ \pm} \mu^{\mp}$ events).

The final selection applied is based on the variable "stransverse mass," $m_{T 2}$, defined as [23,24]

$m_{T 2} \equiv \min _{q_{T}}\left[\max \left(m_{T}\left(\mathbf{p}_{T}^{\ell 1}, \mathbf{q}_{T}\right), m_{T}\left(\mathbf{p}_{T}^{\ell 2}, \mathbf{p}^{\text {miss }}-\mathbf{q}_{T}\right)\right)\right]$, 


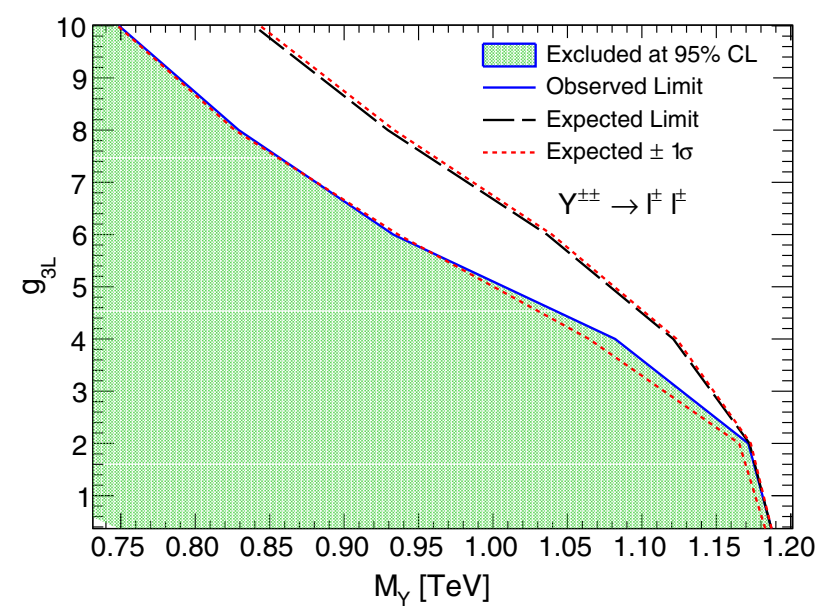

FIG. 8. Excluded parameter space for doubly charged bileptons. The observed and expected limits are shown by the solid blue and long-dashed lines, respectively. The dashed red lines represent the $1 \sigma$ variation on the expected limits.

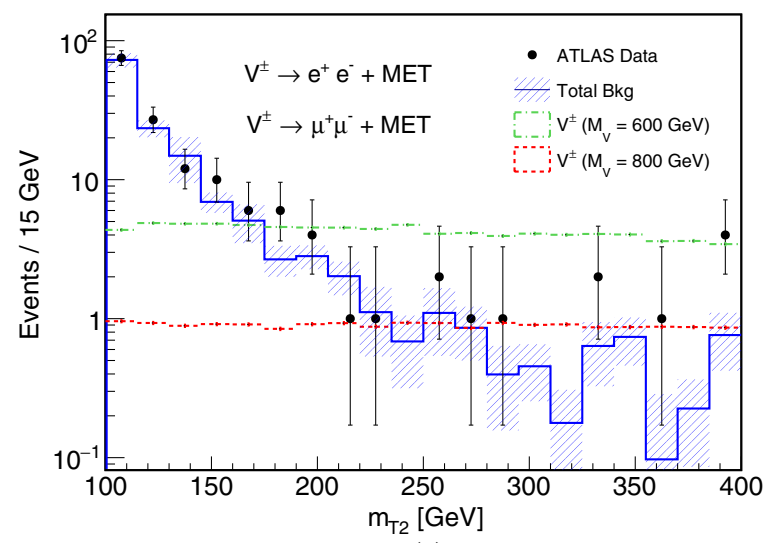

(a)

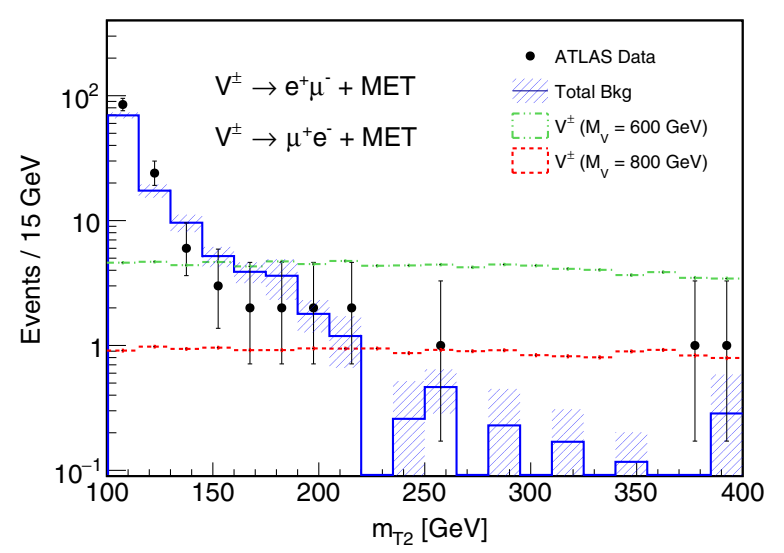

(b)

FIG. 9. $m_{T 2}$ distribution for ATLAS data, total background and two simulated singly charged bilepton signals. The same flavor $m_{T 2}$ distributions (a) includes $e^{+} e^{-}$and $\mu^{+} \mu^{-}$events, while the different flavor (b) corresponds to $e^{ \pm} \mu^{\mp}$ events.

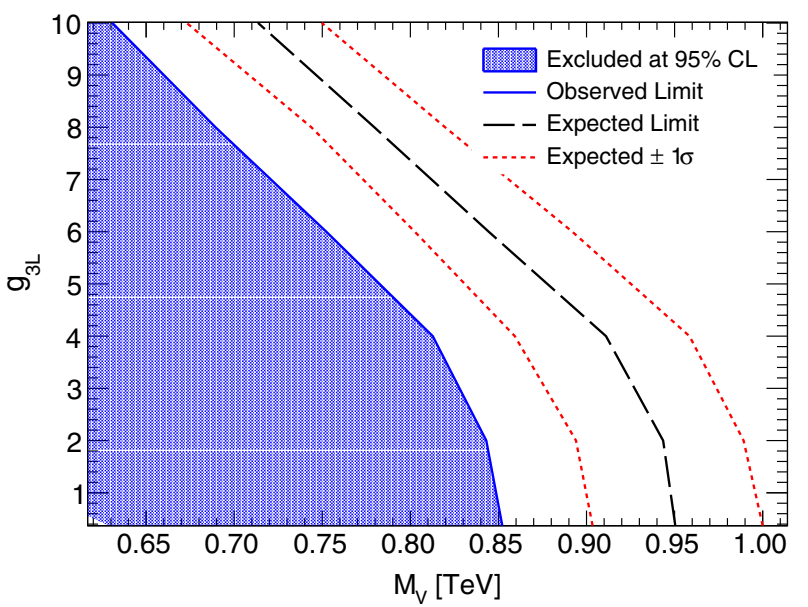

FIG. 10. Excluded parameter space for singly charged bileptons. The observed and expected limits are indicated. The dashed red lines represent the $1 \sigma$ variation on the expected limits.

where

$$
m_{T}\left(\mathbf{p}_{T}, \mathbf{q}_{T}\right)=\sqrt{2\left(p_{T} q_{T}-\mathbf{p}_{T} \cdot \mathbf{q}_{T}\right)},
$$

$\mathbf{p}_{T}^{\ell 1}$ and $\mathbf{p}_{T}^{\ell 2}$ are the two leptons vectors transverse momentum, $\mathbf{p}^{\text {miss }}$ is the missing transverse momentum and $\mathbf{q}_{T}$ is a transverse momentum vector that minimizes the larger of $m_{T}\left(\mathbf{p}_{T}^{\ell 1}, \mathbf{q}_{T}\right)$ and $m_{T}\left(\mathbf{p}_{T}^{\ell 2}, \mathbf{p}^{\text {miss }}-\mathbf{q}_{T}\right)$.

The $m_{T 2}$ variable is used in the statistical analysis where the signal region is defined by requiring $m_{T 2}>100 \mathrm{GeV}$. Figures 9(a) and 9(b) show the $m_{T 2}$ distribution for the selected events in the same flavor and different flavor categories, respectively. Two signal examples are also shown.

The observed and expected exclusion limits are displayed in Fig. 10. Singly charged bileptons with masses between 600 and $850 \mathrm{GeV}$ are excluded at 95\% C.L. The observed limits are smaller than the expected ones due to the data excess observed in the region $m_{T 2}>250 \mathrm{GeV}$, as we can see in Fig. 9. These results are the first limits derived for singly charged bileptons using LHC data.

\section{CONCLUSIONS}

Limits on the mass and couplings of vector bosons from a 331 model are derived using different ATLAS searches at $13 \mathrm{TeV}$ and $36.1 \mathrm{fb}^{-1}$ of data. Three search channels are investigated: dilepton production, four lepton production and dilepton production plus missing transverse energy. These channels are used to derive exclusion limits on $Z_{331}^{\prime}$, doubly charged and singly charged bileptons, respectively. A $Z_{331}^{\prime}$ with mass smaller than $3.7 \mathrm{TeV}$ is excluded. For bileptons, the coupling strength to leptons is varied and it is found that doubly charged bileptons with masses up to 1.2 $\mathrm{TeV}$ are excluded. For singly charged bileptons, the 
maximum bound is $850 \mathrm{GeV}$, and it represents the very first direct limit on singly charged bileptons obtained with hadron collision data. These results improves previous limits on 331 vector bosons and at the moment this paper was written, they are the most stringent limits on these particles.

\section{ACKNOWLEDGMENTS}

This work is partially supported by the Brazilian National Council for Scientific and Technological Development (CNPq) under Grants No. 308494/2015-6 and No. 402846/2016-8.
[1] M. Singer, J. W. F. Valle, and J. Schechter, Phys. Rev. D 22, 738 (1980).

[2] J. W. F. Valle and M. Singer, Phys. Rev. D 28, 540 (1983).

[3] P. H. Frampton, Phys. Rev. Lett. 69, 2889 (1992).

[4] F. Pisano and V. Pleitez, Phys. Rev. D 46, 410 (1992).

[5] R. M. Fonseca and M. Hirsch, Phys. Rev. D 94, 115003 (2016).

[6] B. Meirose and A. A. Nepomuceno, Phys. Rev. D 84, 055002 (2011).

[7] C. Corian and P. H. Frampton, Mod. Phys. Lett. A 34, 1950076 (2019).

[8] M. B. Tully and G. C. Joshi, Phys. Lett. B 466, 333 (1999).

[9] L. Willmann et al., Phys. Rev. Lett. 82, 49 (1999).

[10] B. Dion, T. Gregoire, D. London, L. Marleau, and H. Nadeau, Phys. Rev. D 59, 075006 (1999).

[11] H. N. Long, N. V. Hop, L. T. Hue, and N. T. T. Van, Nucl. Phys. B943, 114629 (2019).

[12] A. E. Cárcamo Hernández, S. Kovalenko, H. N. Long, and I. Schmidt, J. High Energy Phys. 07 (2018) 144.

[13] G. Altarelli, B. Mele, and M. Ruiz-Altaba, Z. Phys. C 45, 109 (1989); 47, 676(E) (1990).

[14] G. Corcella, C. Corian, A. Costantini, and P. H. Frampton, Phys. Lett. B 785, 73 (2018).

[15] A. Belyaev, N. D. Christensen, and A. Pukhov, Comput. Phys. Commun. 184, 1729 (2013).

[16] A. Nepomuceno, B. Meirose, and F. Eccard, Phys. Rev. D 94, 055020 (2016).

[17] T. Sjöstrand, S. Ask, J. R. Christiansen, R. Corke, N. Desai, P. Ilten, S. Mrenna, S. Prestel, C. O. Rasmussen, and P. Z. Skands, Comput. Phys. Commun. 191, 159 (2015).
[18] J. de Favereau, C. Delaere, P. Demin, A. Giammanco, V. Lematre, A. Mertens, and M. Selvaggi (DELPHES 3 Collaboration), J. High Energy Phys. 02 (2014) 057.

[19] B. Meirose and A. J. Ramalho, Phys. Rev. D 73, 075013 (2006).

[20] B. Meirose and A. J. Ramalho, J. Phys. G 36, 095007 (2009).

[21] P. H. Frampton and D. Ng, Phys. Rev. D 45, 4240 (1992).

[22] M. Muhlleitner and M. Spira, Phys. Rev. D 68, 117701 (2003).

[23] C. G. Lester and D. J. Summers, Phys. Lett. B 463, 99 (1999).

[24] C. G. Lester and B. Nachman, J. High Energy Phys. 03 (2015) 100.

[25] A. Caldwell, D. Kollar, and K. Kroeninger, Comput. Phys. Commun. 180, 2197 (2009).

[26] M. Aaboud et al. (ATLAS Collaboration), J. High Energy Phys. 10 (2017) 182.

[27] C. Salazar, R. H. Benavides, W. A. Ponce, and E. Rojas, J. High Energy Phys. 07 (2015) 096.

[28] S. Profumo and F. S. Queiroz, Eur. Phys. J. C 74, 2960 (2014).

[29] Y.A. Coutinho, V. Salustino Guimares, and A. A. Nepomuceno, Phys. Rev. D 87, 115014 (2013).

[30] M. Aaboud et al. (ATLAS Collaboration), Eur. Phys. J. C 78, 199 (2018).

[31] M. Aaboud et al. (ATLAS Collaboration), Eur. Phys. J. C 78, 995 (2018).

Correction: A typographical error appeared in the second unit given in the first sentence of the fourth paragraph of the Introduction and has been fixed. 